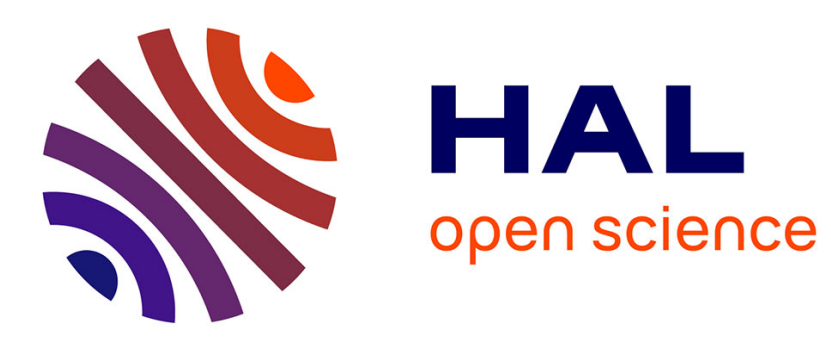

\title{
Morphostructures cantabriques: Picos de Europa, Montaña de León et Palencia (Espagne du nord-ouest)
}

Georges Bertrand

\section{To cite this version:}

Georges Bertrand. Morphostructures cantabriques: Picos de Europa, Montaña de León et Palencia (Espagne du nord-ouest). Revue Géographique des Pyrénées et du Sud-Ouest, 1971, 42 (1), pp.49-70. 10.3406/rgpso.1971.4872 . hal-02570343

\section{HAL Id: hal-02570343 \\ https://hal.science/hal-02570343}

Submitted on 12 May 2020

HAL is a multi-disciplinary open access archive for the deposit and dissemination of scientific research documents, whether they are published or not. The documents may come from teaching and research institutions in France or abroad, or from public or private research centers.
L'archive ouverte pluridisciplinaire HAL, est destinée au dépôt et à la diffusion de documents scientifiques de niveau recherche, publiés ou non, émanant des établissements d'enseignement et de recherche français ou étrangers, des laboratoires publics ou privés. 


\section{Morphostructures cantabriques : Picos de Europa, Montaña de León et Palencia (Espagne du nord-ouest)}

Georges Bertrand

\section{Citer ce document / Cite this document :}

Bertrand Georges. Morphostructures cantabriques : Picos de Europa, Montaña de León et Palencia (Espagne du nord-ouest). In: Revue géographique des Pyrénées et du Sud-Ouest, tome 42, fascicule 1, 1971. pp. 49-70;

doi : https://doi.org/10.3406/rgpso.1971.4872

https://www.persee.fr/doc/rgpso_0035-3221_1971_num_42_1_4872

Fichier pdf généré le 06/04/2018 


\section{Résumé}

Fragment de socle non. granitisé, sédimentaire et souple, où interfèrent les tectogénèses hercyniennes et alpines ; repris par une orogenèse récente (mio-pliocène ?) et vigoureuse (2 $600 \mathrm{~m}$ d'altitude). Compartimentage topographique du versant nord, proche des fosses sous-marines, conforme au jeu tectonique des blocs. Mosaïque en " écailles plissées » du versant sud, davantage solidaire de la Meseta, qui se traduit surtout indirectement par la lithologie. L'articulation des bassins-versants, qui recoupe orthogonale- ment les morphostructures, ne s'explique que par une mise en place synorogénique. Ébauche d'une taxonomie qui situe chaque morphostructure dans un édifice composite, massif ancien repris dans une chaîne de plissement très récente.

\section{Resumen}

Morfo-estructuras cantábricas. (Picos de Europa, Montaña dp. León y Palencia). Fragmento de zócalo sin granitización, sedimentario y blando, en el cual se mezclan las estructuras hercinianas y las alpinas, modelado posteriormente por movimientos orogénicos recientes $O$ mio-pliocenos ?) y de gran intensidad (2 $600 \mathrm{~m}$ de altitud). El recortado topográfico de la vertiente norte, cercana a las fosas submarinas, refleja la interacción tectónica de los bloques. El mosaico de « escamas plegadas » de la vertiente sur, más unido con la meseta, se manifiesta sobre todo en las formaciones líticas. La articulación de las cuencas hidrológicas en relación con la trama morfoestructural depende de una fijación, coetánea de los movimientos orogénicos. Bosquejo taxonómico que sitúa a cada morfoestructura en un conjunto complejo, macizo herciniano parcialmente incluido en una cordillera. 
REVUE GÉOGRAPHIOUE DES PYRÉNÉES ET DU SUDOUEST TOME 42, FASC. 1, pp. 49-70, Toulouse, 1971

\title{
Morphostructures cantabriques :
}

Picos de Europa, Montaña de León et Palencia

(Espagne du nord-ouest)

\author{
par Georges Bertrand*
}

\begin{abstract}
"The Cantabrian mountain chain as it is now exposed at the surface is rather an incongruous structure"...

"Without doubt it is the Mesozoic and Cenozoic history of this part of earth's crust which caused such as unusual picture of a hercynian orogene, Mesozoic emergences, denudations and blanketing and finally Tertiary emergence and sedimentation have defined the present outcrop of the Palaeozoic rocks, events which were to a large degree independent of the original structure".
\end{abstract}

U. de Sitter, 1963 (1).

Le bourrelet montagneux dressé entre l'Océan Atlantique et les "páramos " de Vieille Castille, du méridien d'Oviedo à celui de Santander, n'est pas un relief simple. Large de 60 à $70 \mathrm{~km}$ sur 80 à $90 \mathrm{~km}$ de long, il se présente comme un volume rocheux à la fois compact et compartimenté où s'enchevêtrent massifs, gorges et bassins. Il culmine à $2648 \mathrm{~m}$ dans les Picos de Europa et une bonne douzaine de sommets dépassent $2500 \mathrm{~m}$. Par sa position littorale et son orientation EW, il isole l'Espagne humide et l'Espagne sèche ( $H$. Lautensach). L'organisation interne du relief est dominée par la dissymétrie du massif de part et d'autre de la "divisoria " (ligne de partage des eaux) : sur la façade cantabrique, des pentes fortes et disséquées, des torrents courts à profil longitudinal tendu, un niveau de base proche et déprimé ; sur la façade castillane, des bassins ouverts traversés

* Chargé d'enseignement de géographie, Toulouse.

(1) Les noms d'auteurs suivis d'une date renvoient à la bibliographie in fine. 
par de longues rivières qui coulent encore à $1000 \mathrm{~m}$ d'altitude au débouché de la montagne, les unes vers l'Ebre méditerranéen, les autres vers le Douro portugais. Le Pico de Tres Mares, ou de Tres Aguas, situé dans la partie sud-orientale de la région étudiée, illustre cette divergence hydrographique, indice d'un nœud structural de premier ordre.

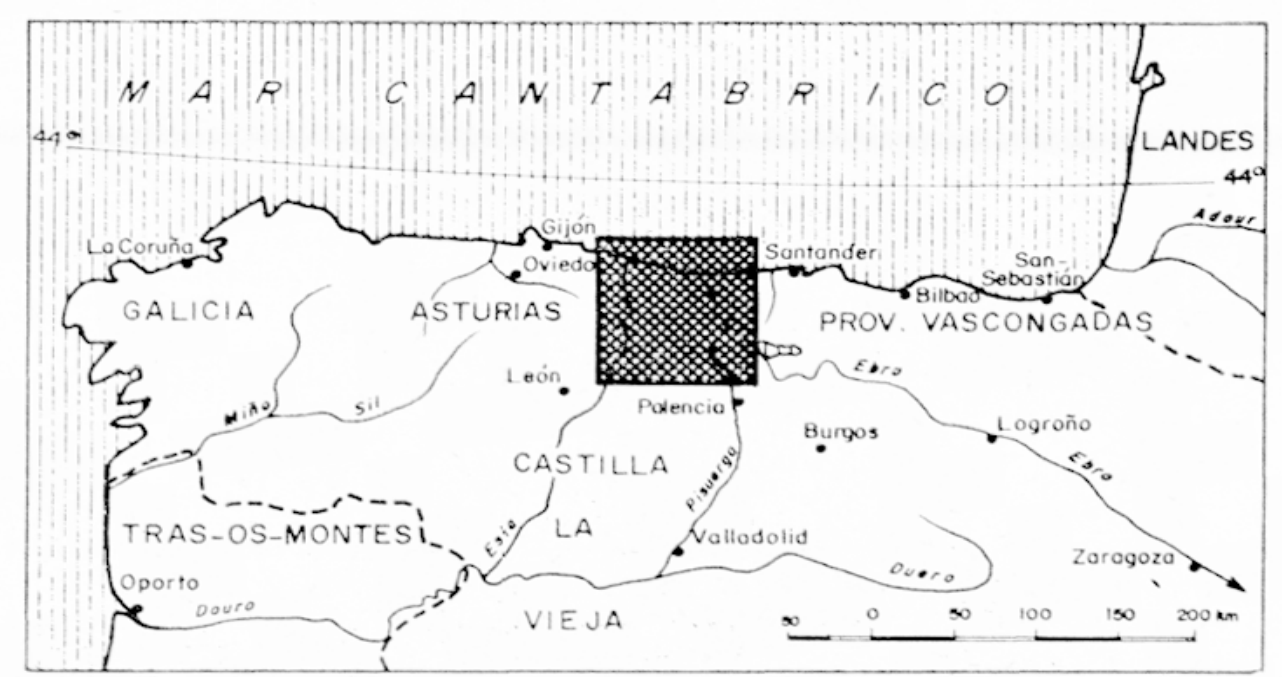

Fig. 1

Les montagnes cantabriques.

Croquis de localisation.

Les montagnes cantabriques centrales (Picos de Europa, Montaña de León et Palencia) correspondent en première analyse à un fragment de zone externe du socle hercynien. Les couches sédimentaires d'âge primaire sont violemment plissées, disloquées, soulevées. L'unité de cet ensemble montagneux est d'abord topographique, c'est-à-dire orogénique, et très secondairement structurale, c'est-à-dire tectogénique.

L'influence de la structure est aussi évidente que malaisée à définir. Le canevas structural, peu visible au niveau de l'architecture élémentaire du versant, se complique quand on passe à l'articulation des grandes unités topographiques et devient insaisissable lorsqu'on essaic de préciser les conditions de la mise en place de l'ensemble montagneux. Cette étude préparatoire a pour but d'analyser les "morphostructures " au sens de I.P. Gue- 
rassimov et de J.P. Mechtcheriakov (2), c'est-à-dire toutes les unités tectoniques supérieures, du massif cantabrique lui-même aux compartiments structuraux d'échelle kilométrique qui en rythment le relief. On laisse momentanément de côté les formes structurales de versant d'échelle hectométrique, dont il ne sera question que de façon très indirecte, ainsi que l'ensemble des reliefs et des modelés climatiques. Cette mise au point résume très brièvement les résultats de plusieurs campagnes réalisées de 1963 à 1967, souvent en collaboration avec les géologues. Elle utilise très largement une bibliographie géologique aussi abondante qu'hétérogène (cf. l'orientation bibliographique in fine) (3).

La prospection géologique a débuté relativement tôt avec les esquisses stratigraphiques de G. Schultz (1837-1878), Ch. Barrois (1882), et l'ébauche tectonique du versant léonais par Casiano de Prado (1861). Une seconde phase de recherches, intense mais désordonnée, aboutit aux premières grandes interprétations structurales (Mengaud, 1910-1939; H. Karrenberg, 1934 ; E. et F. Hernandez Pacheco, 1935 et 1944). R. Ciry publie en 1936 sa magistrale étude des bordures du massif ancien des Asturies, prolongée par les travaux de P. Rat (1959) et de P. Comte (1959). La cartographie géologique ne s'organise qu'à partir de 1945-1950. Sous l'impulsion de N. Llopis-Lladó, les géologues de l'université d'Oviedo étudient le bassin houiller et ses bordures (M. Julivert, 1960 ; J.A. Martínez-Alvarez, 1962, etc.). Une première esquisse géologique à $1 / 100000 \mathrm{du}$ versant castillan est proposée par U. de Sitter et son équipe hollandaise (1965). Elle est parachevée par la publication d'excellentes cartes à $1 / 50000$ (4). Le versant cantabrique, plus complexe, n'est pas entic̀rement cartographié, à part une esquisse structurale sommaire de J.A. Martinez-Alvarez. Les structures du massif des Picos de Europa, compartiment essentiel de l'édifice cantabrique, ne sont pas encore parfaitement débrouillées. Les essais d'interprétation stratigraphique et tectonique ne portent donc que sur un certain nombre de secteurs et ils ne concordent pas toujours. Les auteurs soulignent le caractère provisoire, hypothétique, de leur synthèse. Les recherches en cours risquent de modifier l'image que l'on se fait des structures profondes et même de certaines structures épidermiques. Cette situation explique pour partie le petit nombre de recherches géomorphologiques, d'ailleurs essenticllement consacrées à l'étude des modelés climatiques. Il faut renverser la question. L'analyse géomorphologique des volumes topographi-

(2) J.P. Mechtcherjakoy : Les concepts de morphostructure et de morphosculpture : un nouvel instrument de l'analyse géomorphologique, Annales de Geographie, 1968, 423, pp. 539-552.

(3) Cette étude a été rédigée en 1967-1968.

(4) Toutes les cartes géologiques au $1 / 50000^{\circ}$ de l'équipe de U. de Sitter sont publiées dans la revue Leidse Geologische Medcdelingen, Leiden. 
ques et des formes structurales peut contribuer à une meilleure compréhension de certains phénomènes géologiques, en particulier de ceux qui se rattachent à la tectogénèse et à l'orogénèse.

Cette orientation se matérialise par l'élaboration à partir de critères essentiellement géomorphologiques, d'une carte de morphostructures et d'une planche de coupes géologiques (cf. horstextes).

\section{L'environnement structural}

Les montagnes cantabriques centrales appartiennent à la "Cordillère cantabrique » des géologues et des géographes qui s'étire de la Galice aux Pyrénées. Dans cet ensemble, on distingue traditionmellement, après Dantín Cereceda et E. Hernández Pacheco (1913), deux grandes unités tectoniques.

A l'ouest, la région " astúrica " correspond aux massifs d'Asturies et de León et déborde vers l'est sur la province de Santander jusqu'à la vallée du Nansa. Dans l'ensemble, c'est un voussoir de terrains sédimentaires primaires accolé au socle granitisé de Galice. Le contact avec l'avant-pays galicien s'effectue par la fameuse « rodilla astúrica ", gigantesque virgation des faisceaux plissés hercyniens venus, semble-t-il, se mouler et s'écraser contre le tréfonds cristallin. Les plis, grossièrement orientés EW, s'incurvent et s'arquent selon une direction méridienne à partir de la vallée du Sella.

A l'est, la région «cantábrica " se situe sur l'emplacement du complexe sédimentaire basque (P. Rat, 1959). Des terrains du Secondaire et du Tertiaire, empilés sur de grandes épaisseurs, ont été plissés et soulevés au moment de l'orogénie pyrénéoalpine. Ils recouvrent la presque totalité de la province de Santander et du Pays basque espagnol pour se terminer, à l'est, à la vallée de l'Oria où commencerait la chaîne pyrénéenne. La proximité de la fosse sédimentaire basque se traduit, dans la région étudiée, par la pénétration en golfe de terrains tertiaires et secondaires, coincés dans des bassins d'effondrement ou synclinaux est-ouest qui se rétrécissent jusqu'à disparaître dans la masse des Picos de Europa et des massifs septentrionaux. Le plus vaste correspond au demi-synclinorium de San Vicente-de-la-Barquera. Plus à l'ouest, cet ensemble sédimentaire est relayé par le sillon de Cangas de Onis qui constitue l'extrémité orientale du bassin sédimentaire d'Oviedo. La couverture sédimentaire ne pénètre pas à l'intérieur du versant castillan. Par contre, un liseré de terrains crétacés très plissés, de 1 à $2 \mathrm{~km}$ de large, se glisse entre le massif primaire soulevé et la Meseta. 
Dans leur ensemble, les montagnes cantabriques centrales se rattachent au domaine structural « asturien » puisque leur limite orientale correspond au brusque plongement du socle primaire sous la carapace permo-triasique. Mais elles sont cernées sur trois côtés et assez largement pénétrées sur le versant nord par des éléments de la couverture sédimentairc. Il s'agit donc d'un fragment de massif ancien engagé dans les marges d'une chaîne de plissement. "El pais cantábro-astúrico participa, pues, de dos regiones hispanicas bien distintas : de la España herciniana y de la España alpina; pero, tanto en una como en otra, tiene caracteristicas tan particulares que le dan un sello de rigurosa originalidad dentro de cada una de aquellas grandes unidades » (N. Llopis-Lladó).

Enfin, la « cordillère cantabrique » doit être située dans son environnement structural. Au sud, s'étend le socle primaire de la Meseta. Il est enseveli sous une puissante série détritique oligomiocène plus ou moins déforméc. C'est le piémont sud-cantabrique. Au nord et à $60-70 \mathrm{~km}$ au large des côtes asturiennes, les grandes fosses sous-marines de Las Pregonas s'enfoncent à plus de $4000 \mathrm{~m}$ de profondeur, offrant une sorte de " négatif " du massif cantabrique.

Le problème structural est posé. Qucls sont la part et le style des structures hercyniennes et alpines? Le massif cantabrique est-il un simple « bourrelet marginal " de la Meseta? S'agit-il d'une chaîne $d$ ' "arrière-pays " en train de se fragmenter, de se rétrécir et de disparaître par pans successifs dans l'océan? Quel équilibre isostatique unit les compartiments soulevés aux fosses sous-marines? Peut-on envisager un parallèle avec l'évolution tectogénique et orogénique de la Zone Axiale pyrénéenne? Il est déjà possible d'individualiser le massif cantabrique par rapport aux cordillères centrales ibériques et à la Sierra Morena qui ne sont que de simples voussoirs du socle castillan. Enfin, on voit mieux les causes tectoniques de la dissymé. trie géomorphologique entre la façade castillane, plus ou moins soudée et solidaire de la Meseta, et la façade cantabrique, voisine des fosses océaniques.

\section{Le versant cantabrique : tectonique de blocs et « nappes chevauchantes »}

\section{Le verscant cantabrique}

$35 \mathrm{~km}$ de large pour $2600 \mathrm{~m}$ de dénivelée, s'organisc en compartiments tectoniques orientés Est-Ouest et inégalement soulevés.

a) La banquette littorale de la « Marina "(200-250 m d'altitude sur 1.6 km de large) correspond aux aplanissements étagés 
des "Rasas " calcaires et des "Sierras Planas " quartzitiques. Le socle primaire conserve quelques pincées de la couverture secondaire. A l'est, il s'ennoie assez rapidement sous les terrains sédimentaires du synclinorium de San Vicente-de-la-Barquera qui constitue l'extrême pointe du système structural basco-cantabrique. Les argiles et les grès du Wealdien, les calcaires à Rudistes, les calcaires et les marnes du Cénomanien-Sénonien-Eocène ainsi que les grès bréchoïdes de l'Oligocène supérieur sont largement plissés, sauf le long d'accidents cassants où le Trias a giclé en diapir. Appuyé au sud sur les calcaires primaires, le synclinorium plonge dans l'Océan par l'intermédiaire d'une flexure ou d'une faille flexurée. Plus à l'est, le bloc côtier est bordé par un liseré discontinu de terrains sédimentaires (îlots, Atalaya de Llanes, Cap de Ribadesella) qui jalonnent un important accident tectonique. Escarpement de faille? Flexure "continentale "? C'est tout le problème du rapport tectonique entre le massif montagneux dans son ensemble et les reliefs immergés.

b) La Sierra de Cuera $(500-1000 \mathrm{~m}$ d'altitude sur 2 à $8 \mathrm{~km}$ de large) est un môle classique soulevé entre deux failles et dépouillé de sa couverture sédimentaire. Les plateaux karstiques accidentés de poljés y alternent avec de grands modelés de dissection taillés dans les schistes-quartzites. A l'est du Deva, le massif s'ennoie progressivement sous les couches permo-triasiques qui viennent l'encapuchonner pour former l'anticlinal dissymétrique de l'Escudo de Cabúerniga.

c) Le Sillon prélittoral correspond davantage à une grande cicatrice tectonique qu'à un bloc effondré. Vers l'ouest, s'aligne un chapelet de bassins contenant des lambeaux de couverture plissés ou basculés : synclinal de Cangas de Onís qui prolonge le grand bassin sédimentaire d'Oviedo, fossé tectonique du pays de Cón, pupitre de couches permo-triasiques de la cuvette de Robellada. Au centre, dans la région de Cabrales, on passe à la simple zone de broyage dans la "caliza de montaña " jalonnée par quelques bassins étriqués (Las Arenas, Mier). A l'est, le bassin tectonique de Panes apparaît comme le symétrique de celui de Cangas de Onís. On y trouve le Crétacé et le Nummulitique plissés. Mais ici, les plis sont plus vigoureux, les voûtes anticlinales fermement dessinćes et, à $1 \mathrm{~km}$ à l'est d'Alevia, le môle de la Sierra de Cuera vient s'appuyer sur les couches cénomaniennes pliées en accordéon.

d) Les Picos de Europa, pièce maîtresse du versant nord, sont un puissant massif de "caliza de montaña ", calcaire dinantien cristallin et parfois dolomitique, qui donne un paysage chaotique né de la combinaison de formes karstiques et glaciaires.

Le bloc septentrional, dit du plateau d'Enol, ne dépasse pas $1400-1500 \mathrm{~m}$ d'altitude pour 4 à $10 \mathrm{~km}$ de large. Il est limité au sud par la grande fracture de la Hermida jalonnée de petits bas- 
sins qui se rétrécissent vers l'ouest. A l'est, c'est le demi-synclinal de Puentenansa bourré de grès permo-triasiques et de calcaires noirs du Jurassique. Il se termine en balcon, vers $800 \mathrm{~m}$ d'altitude, au-dessus des gorges du Deva et du bassin de Lebeña. A l'ouest du Deva, la fracture de la Hermida tranche directement les calcaires viséens. Elle est flanquée de lambeaux de Permotrias et de schistes westphaliens (Tresviso et Bulnes).

Le bloc méridional, qui n'a que 2 à $3 \mathrm{~km}$ de large, porte les hauts sommets de la région à plus de $2500 \mathrm{~m}$ et constitue la culmination tectonique des montagnes cantabriques centrales. C'est une haute montagne karstique, fortement englacée au Quaternaire et encore parsemée de névés. Au sud, une muraille de plus de $2000 \mathrm{~m}$ domine la fosse des bassins intramontagnards. C'est l'accident le plus important de la région. Il correspond à une gigantesque faille oblique, peut-être à un chevauchement frontal. La masse rigide des calcaires dinantiens vient écraser et localement rebrousser les couches plus souples des schistes et des grauwackes du Westphalien.

c) Les bassins intramontagnards septentrionaux de Liébana, du Valdeon et du Sajambre. De taille et d'altitude variables, ils se rattachent encore, dans une certaine mesure, à la tectonique de blocs. Leur origine est tectonique bien qu'il s'agisse de bassins largement évidés en roches tendres (faciès Culm).

\section{La tectonique de bloc ne suffit pas à définir le style structural du versant nord}

En plus de ces structures à échelle kilométrique qui rythment le compartimentage topographique du versant cantabrique, il faut considérer les déformations plus souples, responsables des formes structurales hectométriques ou encore plus menues.

On a déjà noté que le socle primaire est entièrement constitué de terrains vigoureusement plissés et que les plis s'orientent généralement d'est en ouest, sauf à l'ouest où ils s'infléchissent brusquement, dessinant une grande concavité ouverte vers le sud. A l'intéricur de cet are, les plis s'organisent en une rigoureuse ordonnance. Presque tous les pendages s'orientent vers le nord, quel que soit le bloc considéré. Le style est isoclinal avec des pendages de l'ordre de 3.5 à 75". Cette uniformité cache en fait des structures particulièrement complexes. Le long des gorges du Cares ou du Sella qui scient entièrement le massif des Picos de Europa, les plis semblent s'ordonner en paquets d'un ou plusieurs kilomètres de large. Les contacts entre deux paquets successifs sont le plus souvent mécaniques avec des étirements et des broyages. Il semble que des plis très serrés, souvent écrasés, soient venus s'appuyer et parfois chevaucher les uns sur les 
autres. Il s'y ajoute un argument stratigraphique. Les calcaires griottes du Viséen, qui forment la base du complexe de la "caliza de montaña ", se retrouvent à plusieurs reprises pincés dans la masse des calcaires gris et bleutés du Dinantien. Ils paraissent marquer des redoublements de couche. On pourrait expliquer ainsi l'épaisseur des affleurements calcaires des Picos de Europa. Les différents paquets de plis isoclinaux constituent autant d' "écailles" ou de "nappes chevauchantes" imbriquées les unes dans les autres.

Ces structures demi-souples inscrites dans le socle primaire ne sont pas sans rapport avec les plissements de la couverture sédimentaire. Ces derniers sont généralement plus doux, les pendages atteignent rarement $30^{\circ}$. Mais on retrouve, dans l'ensemble, le même style isoclinal. Il y a une évidente continuité entre les déformations du socle et celles, plus souples, des terrains sédimentaires. Le cas le plus spectaculaire est celui du pli anticlinal de l'Escudo de Cabúerniga, développé dans le Permo-Trias et le Jurassique, qui se continue dans le calcaire primaire en passant au pli-faille.

\section{Les interprétations données par les géologues sont à la fois grandioses et sommaires}

Pendant un temps, L. Mengaud, avec l'appui de P. Termier et de L. Bertrand, s'est fait le champion de la théorie des nappes de charriage : " la masse calcaire des Picos de Europa appartient à une nappe qui repose nettement sur des marnes d'âge secondaire indéterminé, apparaissant en fenêtres tectoniquess à Lebeña et Campo Mayor, et qui chevauche aussi les schistes du Carbonifère supérieur du "valle" de Liébana »; il explique de la même manière les contacts anormaux des "Sierras Planas" et date ces charriages du «post-nummulitique». En fait, cette hypothèse repose sur une erreur d'observation. Les fameuses fenêtres de Lebaña et de Campo Mayor sont tout simplement des charnières synclinales de schistes carbonifères, fortement pincés par les calcaires dinantiens et déversés vers le sud. L'auteur luimême est revenu sur une interprétation qui n'a d'ailleurs jamais été acceptée par les autres géologues.

Dans son interprétation générale des structures asturiennes, N. Llopis Lladó insiste sur l'autochtonie du massif cantabrique. Il y décèle l'interférence de deux styles structuraux très différents. D'abord, une série de plissements énergiques d'âge hercynien, essentiellement de la phase "asturienne", suivis par une longue période de consolidation. Ensuite, une tectonique de blocs de type "germanique " ( montañas de bloques", "schollen-gebirge »). En se référant au poudingue oligocène faillé de Posada 
étudié par Gomez de Llarena (1934), N. Llopis-Lladó (1950-1964), date les fractures de la tectogénèse "pyrénéenne "; il réduit l'importance des chevauchements et en fait de simples failles obliques, par exemple dans sa coupe du rebord méridional de la Sierra de Cuera (1958).

A la suite de recherches plus poussées, J.A. Martinez-Alvarez propose une interprétation qui, dans une certaine mesure, concilie ces deux positions extrêmes; il souligne l'importance des contacts mécaniques obliques entre les différents blocs tectoniques et insiste sur « la existencia de una megaestructura caracterizada por la presencia de grandes frentes de cabalgamiento " (1962, 1965). Il rejoint ainsi les observations des géologues hollandais (de Sitter, 1963). Ce n'est pas un retour à la théorie des charriages. Les mouvements latéraux sont limités dans l'espace et se développent au sein d'une masse rocheuse enracinée. On peut parler de chevauchements frontaux, ou mieux, de nappes chevauchantes ( mantos cabalgantes ») (J.A. Martínez-Alvarez, 1965).

Le versant cantabrique apparaît donc comme une région de tectonique vigoureuse où interfèrent les formes de fracturation et de plissement, avec deux phases majeures du point de vue de la tectogénèse, l' " hercynienne » et peut-être, surtout, la "pyrénéenne ". C'est, en effet, à cette époque que les écailles chevauchantes se sont mises en place, incorporant la couverture sédimentaire dans un système structural composite où les déformations souples, tout en s'amortissant assez rapidement, ont cependant remanié un "socle" primaire resté relativement plastique. L'examen du versant sud confirme partiellement cette hypothèse.

\section{Le versan't castillan : « les écailles plissées 》}

\section{Le versant castillan}

$1000-2500 \mathrm{~m}$ d'altitude sur 20 à $40 \mathrm{~km}$ de large, est une mosaïque de massifs et de bassins aux contacts complexes.

a) Le " massif central "Peña Prieta-Curavacas-Orpiñas est davantage une unité topographique que structurale. Il correspond à une partie de la "divisoria " cantabrique. L'élément structural prépondérant est la "formation de Curavacas", puissant synclinorium de conglomérats westphalien à ciment hématiteux, qui repose en discordance sur les terrains plus anciens. Son rebord méridional aux hautes parois gris métallique porte, à $2520 \mathrm{~m}$, le point culminant du versant sud. 
b) Les sierras calcaires occidentales (Mampodre, Coriscao) et méridionales (Sierra del Brezo, Peña Corada) s'individualisent par leurs formes structurales vigoureuses. Elles se rattachent à deux ensembles stratigraphiques différents. D'une part, les calcaires du Westphalien qui alternent avec de larges assises de schistes et de grès, ce qui favorise à la fois les dysharmonies et les érosions différentielles; d'autre part, la "caliza de montaña " qui sans atteindre la compacité des calcaires des Picos de Europa peut constituer des ensembles massifs mais riches en formes structurales de détail. Les structures épidermiques sont généralement "bouillonnées", plis et fractures s'enchevêtrent pour donner des reliefs de type appalachien (synclinaux perchés, crêtes, barres etc.).

c) Les bassins intramontagnards méridionaux. Entre les môles résistants constitués par les massifs calcaires et conglomératiques, s'étendent des bassins évasés qui ont été déblayés dans des schistes et des grès du Westphalien et du Stéphanien conservés en position synclinoriale. Toutes ces roches tendres ont été tranchées et aplanies au moment de la mise en place des rañas qui, contrairement à ce qui a été parfois affirmé, s'enracinent profondément à l'intérieur de la masse soulevée du massif ancien.

d) Les massifs permo-triasiques du rebord oriental. La couverture permo-triasique formée par un conglomérat siliceux très résistant individualise un certain nombre de reliefs structuraux : parois verticales des crêts bordiers (Peña Labra), tables et pupitres parfaitement rigides (Valdecebollas, Tresmares). Elle représente aussi l'un des rares repères de l'évolution géomorphologique puisqu'elle jalonne la "surface" post-hercynienne dont on peut suivre ainsi les déformations sur toute la bordure orientale. "Le Massif asturien s'ennoie vers l'est en dessinant un certain nombre d'anticlinaux de fond, dissymétriques, en genou vers le sud. Entre ces anticlinaux s'engagent des langues synclinales de revêtement "(Ciry, 1936). Cette disposition souligne parfaitement l'importance de la tectogénèse post-hercynienne. C'est en partant de ces déformations marginales que l'on peut suivre les accidents qui pénètrent à l'intérieur du socle. Le plus important prend en diagonale tout le versant sud par les bassins de Camporredondo et de Riaño. Cette orientation réapparaît sur la bordure méridionale avec le décrochement oriental de la Sierra del Brezo. On ne retrouve donc jamais la tectonique de blocs si évidente sur le versant nord.

e) Le contact avec les hautes plaines de Castille s'opère par l'intermédiaire d'une faille est-ouest qui tranche indifféremment les masses calcaires des Sierras méridionales et les schistes carbonifères évidés. Cet accident de première grandeur dénivelle définitivement le socle. Deux unités structurales très différentes se succèdent du côté affaissé. 
Au pied du massif ancien, s'étire une bande de 1 à $2 \mathrm{~km}$ de terrains sédimentaires crétacés plissés et fracturés : graviers et sables kaoliniques, grès et surtout complexe calcaire du Turonien au Maestrichtien dans lesquels se sculptent plusieurs lignes de crêts. D'après G. de Llarena (1934) et H. Karrenberg (1934), le Crétacé, qui plonge presque toujours en direction du nord, serait chevauché par les terrains carbonifères et chevaucherait lui-même les conglomérats. Sans nier cette interprétation, on peut, après R. Ciry (1936), en limiter la signification structurale. " Le Paléozoïque se montre enraciné ct affecte unc disposition anticlinale avec tendance au déversement vers le sud. Le Crétacé vertical ou même plongeant légèrement vers le nord, qu'on suit au pied du massif, correspond à la couverture normale de celui-ci et représente la retombée de l'anticlinal... L'ampleur des mouvements au sud est assez réduite et nulle part je n'ai observé de chevauchements de quelque importance ". Cette bande de terrains crétacés s'évase à l'est de Cerverade-Pisuerga où elle affleure en même temps que des calcaires jurassiques et des marnes gypsifères à ophites du Keuper. C'est le " pays plissé " de R. Ciry, compliqué par des décollements diapiriques au niveau du Keuper qui donnent des structures en écailles avec de petits charriages. Ces structures sont déjà étrangères au massif primaire cantabrique et se rattachent à la fois au Pays Basque et aux Monts Celtibériques.

En concordance ou en légère discordance avec cet ensemble crétacé plissé, un puissant conglomérat d'au moins $1000 \mathrm{~m}$ d'épaisseur se développe vers le sud. C'est une formation synorogénique de piémont d'âge présumé éo-oligocène. Elle a été étudiée par A.J. Pannekoek (1959) qui en a donné une première ébauche stratigraphique : à la base, un conglomérat constitué presque exclusivement par des éléments roulés de calcaire crétacé, ce qui laisserait supposer que la couverture crétacée a été beaucoup plus étendue qu'actuellement et qu'elle a été démantelée au moment des premiers mouvements dits "pyrénéens"; quand on s'élève dans la formation, les blocs de quartzite deviennent de plus en plus nombreux et passent à des graviers quartzeux qui alternent avec des sables et des argiles rouges. A mesure qu'on s'éloigne de la bordure montagneuse, en direction du centre de la cuvette léonaise, ce complexe détritique grossier passe latéralement à des marnes blanches fossilifères, datées du Miocène, et à des évaporites. En appliquant le principe de la sédimentation inverse, on note que le massif primaire est déjà largement démantelé à la fin du Miocène. Les mouvements tectoniques se sont poursuivis au moins jusqu'à cette époque puisque ces conglomérats sont plissés, redressés à la verticale au contact du socle. Cependant, si les plis sont relativement vigoureux dans les dépôts les plus anciens (ćocènes et oligocènes), ils s'amortissent assez rapidement dans les conglomérats miocènes qui sont, il est vrai, plus éloignés du massif ancien, ce qui réduit la portée de cette observation. 


\section{La prédominance d'un style structural " semi-souple "}

Les bordures sédimentaires mises à part, on peut affirmer que les accidents cassants d'échelle kilométrique sont peu nombreux. On ne peut pas parler de tectonique de blocs. Par contre, le style souple ou semi-souple domine. Sur ce point, les interprétations des géologues concordent (U. de Sitter, P. Comte, M. Julivert, J. A. Martínez-Alvarez).

Les unités structurales majeures sont données par des écailles ( " thrustsheets " de U. de Sitter) de plusieurs kilomètres de largeur, inclinées vers le nord et qui se chevauchent sur leur flanc sud. De part et d'autre d'une ligne allant de Cervera-de-Pisuerga au sud du Mampodre, en passant au nord du lac de Camporredondo et au sud de Riaño, et qu'il appclle la "León Line », U. de Sitter distingue deux ensembles géologiques différents :

- au nord, les "Asturides" constituces exclusivement de Carbonifère et de Dévonien. Cette unité correspond en gros aux bassins intramontagnards septentrionaux et au "massif central». Les principales écailles sont celles des puertos de San Isidro, du Carrión (qui comprend la formation de Curavacas), etc.;

- au sud, les "Leonides» correspondent aux Sierras calcaires méridionales (Peña Corada, Peña Cueto, Sierra del Brezo) qui sont autant d'écailles plus ou moins indépendantes, et aux bassins carbonifères (Sabero, Cea, Guardo).

Ces divisions géologiques n'ont pas toujours un intérêt géomorphologique évident car, le plus souvent, les structures épidermiques de détail, c'est-à-dire la nature et l'agencement des roches à l'intérieur des différentes écailles, l'emportent sur ces mégastructures souvent confuses. Toutes ces écailles sont plissées en structures synclinoriales avec d'importants effets de serrage et d'étirement des couches. Les pendages sont généralement forts, mais moins régulièrement que sur le versant cantabrique; de même, les plis ne sont pas isoclinaux bien que les pendages nord soient les plus fréquents.

Ces structures en "écailles replissécs" (P. Comte, 1959) sont, selon toute vraisemblance, dessinées au moins depuis la phase de tectogénèse « asturienne ». Cependant, quand on se rapproche de la bordure orientale, on se rend compte que si les structures post-hercyniennes, représentées par les déformations semi-souples de la couverture permo-triasique, recoupent indifféremment les axes structuraux hercyniens, elles ont profondément déformé le socle : d'abord, en le dénivelant en plis-failles dont la flèche dépasse le kilomètre; ensuite, en favorisant un déversement général des plis vers le sud; enfin, en s'accompagnant d'effets de 
serrage. "Au cours de l'orogénèse tertiaire, comme durant les plissements carbonifères, le style souple a nettement dominé dans les terrains anciens de toute la partie asturico-lćonaise. " ( $P$. Comte).

\section{Mise en place des morphostructures}

\section{La tectogénèse : l'imbrication des structures hercyniennes et alpines}

Les deux phrases de U. de Sitter placées en épigraphe, résument la pensée des géologues de diverses origines qui ont travaillé dans les montagnes cantabriques. Il faut une fois pour toutes renoncer au schéma classique d'une chaîne de montagne plissée à l'époque hercynienne, consolidée au Secondaire puis arasée, fracturée et soulevée au Tertiaire. Comme l'avait depuis longtemps reconnu H. Karrenberg (1934), l'évolution post-hercynienne est en grande partic responsable du style structural actuel.

II semble possible de dater approximativement la dernière phase tectogénique. Sur le versant cantabrique, les dernières couches incorporées au plissement sont d'âge lutétien. Les terrains plus récents et particulièrement l'Oligocène supérieur (niveau à Nummulites intermedius et Lépidocyclines) sont seulement ployés. Sur le versant castillan, les repères chronologiques sont beaucoup moins sûrs. Les plissements s'amortissent dans les conglomérats mal datés du piémont. La formation de base considérée comme éo-oligocène est plissée, alors que les dépôts miocènes, bien datés, sont seulement gondolés ou gauchis. La dernière phase de tectogénèse est donc contemporaine du "plissement pyrénéen ». C'est un des rares points communs avec les structures de la chaîne des Pyrénées.

Les montagnes cantabriques centrales correspondent à un secteur de massif hercynien resté relativement souple du fait de l'absence de masses granitiques et repris, sur ses marges, dans la terminaison du "géosynclinal " basque. Les rapports entre la tectogénèse hercynienne et la tectogénèse tertiaire sont délicats à établir. On peut cependant avancer quelques faits assez bien contrôlés :

- la tectonique de blocs et les grandes fractures qui recoupent en oblique les axes structuraux hercyniens et tranchent la couverture sédimentaire sont sans discussion, tertiaires, même s'il s'agit de vieilles cicatrices primaires qui ont rejoué ;

- les grandes nappes chevauchantes et les écailles ont, elles aussi, dû fonctionner vers la fin du Tertiaire puisque les couches 
primaires chevauchent en plusieurs endroits la couverture prémiocène. La tendance générale au déversement des plis vers le sud sur le versant cantabrique, vers le nord (avec des exceptions) sur le versant castillan date, pour les mêmes raisons, de cette époque ;

- les déformations à la fois souples et brisantes enregistrées par la couverture permo-triasique et jurassique montrent que l'ensemble de l'édifice a été de nouveau plissé au Tertiaire le long d'axes EW ou NE-SW;

- à une échelle plus réduite, les géologues admettent que les plis hercyniens ont dû rejouer au Tertiaire : serrage et écrasement des couches, accentuation des pendages. "Le faisceau de plis hercyniens dont l'édification s'était achevée au Permien avait... la forme d'un arc dont la concavité était orientée vers l'est. Les poussées tertiaires agissant en direction méridienne ont fortement resserré cet arc, et les plis ou plis-failles situés du côté de la concavité ont répondu à la compression longitudinale (conséquences nécessaires du raccourcissement du côté interne que réclame le ploiement de l'arc) par des inflexions et surtout par des oscillations verticales très accentuées de leurs axes. Il est toutefois probable que ces déformations axiales ont été en partie amorcées dès le Carbonifère » (P. Comte, 1959).

\section{L'orogénèse : des volumes montagneux récents}

La surrection de la chaîne n'est pas contemporaine de la dernière phase de tectogénèse. Les volumes montagneux sont beaucoup plus récents que les structures. C'est du moins ce qui ressort d'un examen rapide des formations corrélatives de piémont.

Le versant castillan fournit sur ce point de précieuses indications. La subsidence de la Meseta a débuté à l'Eocène et s'est poursuivie pendant tout le Miocène. Comme on l'a déjà noté, le massif cantabrique a dû commencer à s'individualiser à l'éooligocène. C'est à cette époque que la couverture crétacée a été démantelée et incorporée dans les conglomérats du piémont. Au Miocène, l'apparition de plus en plus fréquente de galets de quartzite montre que le socle est à son tour attaqué (Pannekoek, 1959). On peut considérer que c'est l'acte de naissance du bourrelet montagneux actuel. Le bourgeonnement a dû continuer pendant tout le Miocène. Le mouvement a-t-il persisté pendant le Pliocène? Seule une étude géomorphologique détaillée pourrait donner une réponse. Pour l'instant, il suffit de savoir que la surface des "rañas", qui tronque indifféremment les conglomérats du piémont, les plis de la couverture crétacée et certains affleurements tendres du socle primaire, n'est que faiblement déformée. Il y a, dans l'ensemble, continuité topographique entre 
les évidements intramontagnards et les paramos castillans. A partir du Pliocène, il n'y a donc pas eu de mouvement d'individualisation du versant sud par rapport à la Meseta. Les deux blocs sont restés soudés. S'il y a eu un soulèvement postérieur, il ne peut donc être que de type épéirogénique.

Le versant cantabrique ne fournit aucun dépôt daté postérieur à l'Oligocène. Les formations synorogéniques de piémont sont vraisemblablement novées dans l'océan. Par contre, on possède un certain nombre d'arguments géomorphologiques fournis par le littoral. Il s'agit d'une côte de faille qui dénivelle une surface d'érosion de piémont $(110-120 \mathrm{~m}$ d'altitude $)$ jalonnée par des placages de quartz roulés et que l'on peut dater du Plio-Villafranchien. La fraîcheur de tous les grands escarpements qui limitent les falaises marines et les différents blocs tectoniques laisse supposer par ailleurs qu'on se trouve en présence d'accidents très récents. Sans qu'il soit possible pour le moment de le démontrer de façon irréfutable, nous pensons que l'individualisation tectonique du versant cantabrique est un fait pliocène ou même quaternaire, la surrection différenciée des compartiments montagneux étant compensée par l'effondrement des blocs sous l'océan de part et d'autre d'une sorte de gigantesque flexure continentale dont la charnière se situerait un peu au large des falaises marines actuelles. La proximité des fosses océaniques de Las Pregonas semble corroborer cette hypothèse. On peut même se demander si l'axe tectonique de la cordillère cantabrique, localisé sur les Picos de Europa, ne se déplacerait pas vers le sud. Le versant cantabrique jouerait alors le rôle d'une face interne d'arc montagneux qui se fragmenterait dans l'océan, comme le laisse supposer l'étude géomorphologique détaillée du bloc littoral. Toutefois l'étude des anomalies de la pesanteur n'est pas suffisamment poussée pour qu'on puisse se faire une opinion définitive. La carte gravimétrique d'Espagne souligne l'équilibre tectonique d'ensemble du secteur cantabrique (5). Il faudrait donc admettre, avec P. Birot (1956), que les transferts profonds de matières sont terminés ainsi que les compensations isostatiques, et que l'affaissement du bloc littoral n'est que la manifestation d'un réajustement tardif en période de détente. La dissymétrie tectogénique et orogénique entre les deux versants n'en serait que plus éclatante.

\section{Rapport des morphostructures avec les volumes montagneux et les bassins-versants}

La place des morphostructures dans la définition des relicts cantabriques ne pourra être définitivement précisée que lorsque 
seront connues les "morphosculptures" équivalentes (5). Ces dernières seront l'objet d'une mise au point ultérieure qui portera essentiellement sur les mécanismes d'évidement de la masse montagneuse (aplanissements de type " rañas », érosions glaciaire et torrentielle, etc.). Pour le moment, il suffit de retenir un certain nombre de situations et de problèmes géomorphologiques.

Le fait morphostructural le plus caractéristique repose sur une contradiction au moins apparente dans l'agencement du relief : d'une part, une coïncidence remarquable entre le canevas structural et la distribution des volumes montagneux; d'autre part, l'inadaptation des grands bassins-versants aux compartiments tectoniques.

\section{La conformité d'ensemble entre le massif cantabrique central et les phénomènes tectoniques qui l'ont individualisé}

A l'échelle III de Cailleux-Tricart, le relief traduit presque directement le jeu des mégastructures : socle de la Meseta, voussoir cantabrique, fosses sous-marines de Las Pregonas. Le bloc montagneux s'inscrit entre deux accidents bordiers qui déterminent directement les contacts topographiques : "flexure " littorale au nord, escarpement tectonique au sud. Les rapports entre le volume rocheux soulevé, le volume rocheux déblayé par les érosions successives et le relief actuel ne pourront être abordés que lorsqu'on aura précisé les processus de l'évidement de la montagne et de la mise en place des formations corrélatives du piémont castillan.

2. La conformité d'ensemble entre le jeu tectonique des blocs et le compartimentage topographique du versant cantabrique

Les compartiments topographiques majeurs correspondent directement aux mouvements de différenciation tectonique. Les escarpements qui les limitent sont sans exception des escarpements de faille directs, à peine émoussés et d'autant mieux conservés qu'ils sont généralement taillés dans la « caliza de montaña ». Le meilleur exemple est celui du grand talus de 300 à $600 \mathrm{~m}$ de commandement qui dénivelle le bloc à demi effondré de La Marina par rapport au bloc soulevé de La Sierra de Cuera. Les grands massifs (Picos de Europa, Sierra de Cuera)

(5) A. Rey Pastor : Mapa sismotectónico de la Península Ibérica, 1/2000 000. Inst. Geol. y Min. de Esp. Pour une fréquence sismique de 112 à Lisbonne, on a 0,4 pour la Meseta et 2 pour l'ensemble de la région cantabrique. 
correspondent donc à des horst plus ou moins complexes. Les grands bassins sont tous des bassins d'effondrement où sont conservés des dépôts plus récents ployés en synclinaux (Cangasde-Onís, Puenténansa, Liébana, etc.). Les petits alvéoles intramontagnards ont aussi une origine tectonique (Bulnes, Sotres, puertos de Aliva dans le massif occidental des Picos de Europa, poljé de Llamigo dans la Sierra de Cuera). Cependant, les topographies en creux ne résultent pas toujours entièrement du jeu des blocs. Les fossés, généralement bourrés de roches tendres, ont été partiellement excavés par les érosions successives (collines de dissection de La Liébana, train de crêts dans les dépôts wealdiens de San-Vicente-de-la-Barquera (Marina orientale). Parmi les exceptions, il faut placer les grands entablements de conglomérats permo-triasiques qui forment, entre 1600 et $2000 \mathrm{~m}$ d'altitude, la charpente montagneuse du secteur oriental. La permanence des traits acquis au moment de la mise en place tectonique résulte d'une part du caractère récent des mouvements d'individualisation des blocs, d'autre part du contraste lithologique entre les remplissages tendres des bassins (schistes, faciès wealdien) et les calcaires cristallins qui constituent l'ossature sinon la masse totale des horst (résistance mécanique et "immunité " karstique des escarpements de faille).

3. La conformité entre les morphostructures et les volumes montagneux est moins nette sur le versant castillan. De plus, elle est presque toujours indirecte

Le jeu simple de blocs soulevés et affaissés s'efface ici devant une imbrication d'écailles entourées de multiples esquilles rocheuses. La différenciation des compartiments rocheux s'effectue par l'intermédiaire de la lithologie. Les masses synclinoriales de conglomérats quartzitiques (Curavacas) ou de calcaires compacts (Espigüete) se dégagent des bassins en roches tendres (schistes à faciès Culm des bassins intramontagneux) au même titre que les structures anticlinoriales des calcaires du Mampodre ou des Peñas de Riaño. Les aplanissements liés aux rañas ne mordent jamais sur les massifs en roches dures dont certains se dressent comme des îles au milieu des secteurs de plateaux (Espigüete).

\section{La " divisoria " cantabrique}

La ligne de partage des eaux entre le versant asturo-santanderin et le versant castillan, est, du moins en apparence, indifférente à ce schéma morphostructural ainsi qu'à la distribution des volumes montagneux. 
Le massif cantabrique central présente deux culminations qui sont à la fois tectoniques et topographiques. La plus élevée et la plus continue correspond à l'échine méridionale des Picos de Europa $(2500-2600 \mathrm{~m})$. Elle se situe donc au milieu du versant nord. La seconde, moins élevée et surtout discontinue, occupe une position presque identique en plein versant sud (Mampodre, Sierras de Riaño, Espigüete, Curavacas, Tresmares, Valdecebollas entre 2000 et $2500 \mathrm{~m}$ ).

La "divisoria" hydrologique occupe une situation intermédiaire. Elle est établie sur une dorsale montagneuse appuyée sur quelques massifs entre 2000 et $2500 \mathrm{~m}$ (Coriscao, Peña Prieta, Peña Labra), mais aussi ouverte par de vastes "puertos" en roches tendres qui s'abaissent jusqu'à $1400-1500 \mathrm{~m}$ (Pontón. Pandetrave, etc.). Quelques captures hydrographiques de détail, essentiellement dues aux phénomènes de diffluence et de transfluence du maximum glaciaire, expliquent localement l'apparente "inorganisation" de certains réseaux torrenticls. La fameuse concurrence de drainage au prolit du versant cantabrique, si souvent évoquée dans la littérature géographique, ne joue pas de rôle essenticl dans la localisation de la "divisoria " qui est restée dans son ensemble très stable depuis le début du Quaternaire.

\section{Les relations entre le compartiment morphostructural et l'arti- culation des bassins versants sont complexes}

Les torrents qui divergent du faîte hydrologique recoupent tous les ensembles morphostructuraux en sculptant d'impressionnantes gorges dans les massifs de calcaires et de conglomérats. Le Deva, le Cares et le Sella tranchent les Picos de Europa sur un millier de mètres d'épaisseur et sur une vingtaine de kilomètres de longueur. La même disposition se retrouve sur le versant sud mais elle est moins accentuée du fait de l'extension des bassins en roche tendre et du creusement moindre des cours d'eau. L'ampleur des bouleversements tectoniques ne permet pas d'envisager l'installation du réseau hydrographique à partir d'une surface d'érosion ou d'une couverture sédimentaire discordante. Par contre, il apparaît que tous les bassins-versants sont axés sur un ou plusicurs fossés d'effondrement en roche tendre (ex. : le Deva avec les bassins de Liébana, Lebeña, La Hermida et Panes). Les massifs intermédiaires sont franchis grâce à l'utilisation de relais transverses de fractures et de zones de broyage. Sur plus d'un kilomètre de longueur, la gorge du Cares correspond à une "faille-diaclase "subverticale de $200 \mathrm{~m}$ de hauteur sur 4 à $10 \mathrm{~m}$ de large.

Les morphostructures sont bien la trame des reliefs cantabriques, mais une trame vivante dont il faut envisager, au moins 
à titre d'hypothèse, les relations dynamiques avec les grandes phases de géomorphogénèse. L'organisation de l'écoulement torrentiel et la mise en place des bassins-versants ne paraissent "aberrantes" que si l'on raisonne en fonction du plan structural actuel et dans des conditions de stabilité tectonique. L'évidement de la masse montagneuse et la mise en place du système de drainage ne sont-ils pas, pour l'essentiel, des phénomènes synorogéniques qui ne seraient liés que secondairement aux variations bioclimatiques?

\section{VI. « Plan » structural et taxonomie morphostructurale}

Le tableau ci-après rassemble les donnćes du compartimentage structural en fonction de l'échelle spatiale et des styles structuraux. Chaque morphostructure est replacée dans son environnement tectonique, c'est-à-dire en tenant compte à la fois des unités structurales supérieures auxquelles elle appartient et des unités inférieures qu'elle contient. Cette classification est directement inspirée de celle de A. Cailleux et J. Tricart bien qu'elle introduise deux niveaux spatiaux supplémentaires aux classes III et IV (6). Les discontinuités retenues et le contenu des différentes unités restent cependant de même nature. Enfin, on a établi à titre provisoire un parallèle avec des unités géographiques globales (7).

Ce découpage de l'espace géographique veut être le point de départ d'une méthode de cartographie et de recherche pour des études géomorphologiques ultérieures. "La classification taxonomique est génétique. Elle constitue un instrument pour clarifier les rapports de causalité entre faits différents " (6). La définition des morphostructures est liée à l'échelle des volumes et des mécanismes en cause. A l'intérieur d'une même unité (Picos de Europa, Curavacas, etc.), le style structural change avec l'échelle. La distinction traditionnelle entre des "structures épidermiques " et des "structures profondes" ne suffit plus. Il faut la remplacer par une taxomanie spatiale (et autant que possible temporelle) qui peut seule traduire objectivement l'articulation et la dynamique d'un ensemble structural aussi complexe que le massif cantabrique central.

Enfin, les morphostructures ne doivent pas être uniquement analysées dans une perspective géomorphologique. Elles sont l'un des éléments essentiels de l'organisation de l'espace géographique et constituent la mosaïque de base des paysages cantabriques.

(6) J. Tricart : Principes et méthodes de la géomorphologic, Paris, 1965. p. 90 sqq.

(7) G. Bertrand : Ecologie de l'espace géographique. Recherches pour une science du paysage, C.R. Soc. Biogeographie, Paris, 1970. 
TAXONOMIE MORPHOSTRUCTURALE

Meseta de Vieille Castille ...... Golfe de Gascogne ............ Golfe de Gascogne ..............

Massif Cantabrique Central

Versant Cantabrique (Nord) .

Versant Castillan (Sud) .........

Compartiments du Versant Cantabrique

Compartiments du Versant Castillan ...................

Unités structurales simples

Formes structurales de versant.

Formes structurales élémentaires.

Structures microscopiques
DEFINITIONS MORPHOSTRUCTURALES

socle hercynien et sa couverture détritique cordillère (chaîne de plissement tertiaire) reliefs sous-marins et fosses (Las Pregonas)

orogène. Massif ancien soulevé, déformé, partiellement réincorporé dans une chaîne de plisse ments tertiaire.

tectonique de blocs et nappes chevauchantes système d' " écailles plissées "

môles et fossés (ex. : Sierra de Cuera, Picos de Europa, Liébana)

"écailles " (ex. : Espigüiete, bassin de Riaño)

ex. : synclinal perché, escarpement de faille ex. : crêt, barre, corniche... ..

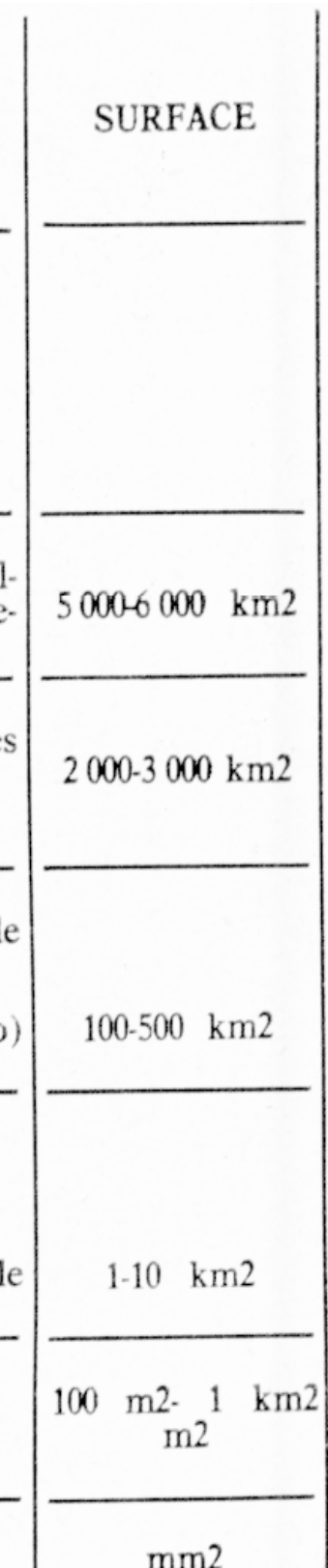

$\mathrm{mm} 2$
CLASSIFICATIONS

TAXONOMIQUES

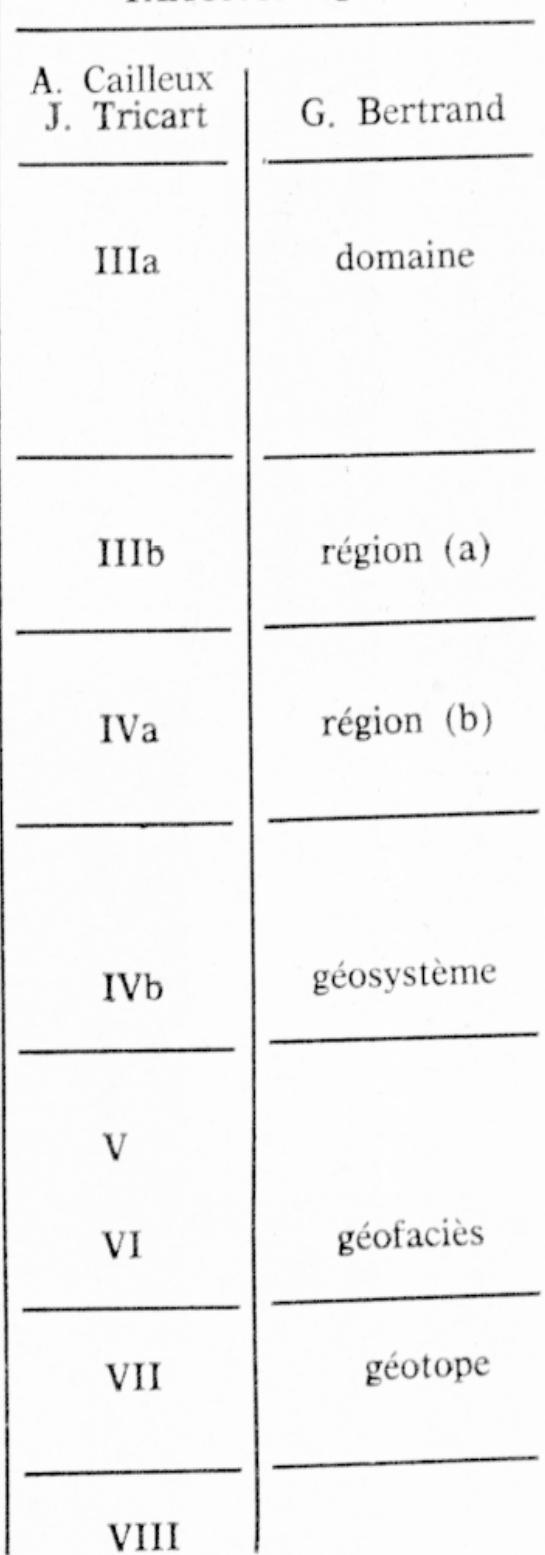




\section{ORIENTATION BIBLIOGRAPHIQUE}

Dans une bibliographie riche de plus de 700 références. on n'a retenu que les auteurs et les études qui ont contribué à faire progresser les problèmes structuraux. J. A. Martínez-Alvarez publie dans le suplemento de Ciencias du Boletín I.D.E.A. (Instituto de Estudios Asturianos) une bibliographie géologique exhaustive qui couvre l'ensemble du secteur cantabrique.

Ch. Barrois, 1882 : Recherches sur les terrains anciens des Asturies et de la Galice, Thèse Sciences, Lille, p. 630.

P. BIRot, 1956 : La Méditerranée et le Moyen-Orient, t. I.

CASIANo dE PRADO, 1861 : Mapa geológica estratigrafica de las montañas de la provincia de Palencia $(1 / 400000)$. Com. estadistica general del Reino. (Sans autres références, Bibl. Instit. Geol. de Madrid).

R. CIRY, 1936 : Etude géologique d'une partie des provinces de Burgos, Palencia, León, Santander. Thèse Sciences, Toulouse. (Bull. Soc. Hist. Nat. Toulouse, t. LXXIV).

P. Comte, 1959 : Recherches sur les terrains anciens de la Cordillère cantabrique, Thèse Sciences. (Mem. Inst. Geol. y Minero de España, t. LX).

J. Gomez de Llarena, 1934 : Algunos ejemplares de cobijaduras tectónicas terciarias en Asturias. León y Palencia, Bol. Soc. Esp. de Historia natural, t. XXXIV, pp. 123-127, Madrid.

J. HAzera, 1968 : La région de Bilbao et son arrière-pays. Etude géomorphologique.

E. Hernandez Pacheco, 1913 : Datos respecto a orogenia de Asturias, Bol. Soc. Esp. de Hist. nat., t. XIII, pp. 143-147.

E. Hernandez Pacheco, 1935 : Discusión de la nota de los Sres Hernandez Pacheco E. y F. Carte geológico del extremo oriental de Asturias, Ibidem, t. XXXV, pp. 487-497.

F. Hernandez Pacheco, 1944 : Nueva hipotesis de la formación tectónica de los Picos de Europa, Invest. $y$ Progreso, t. XV, pp. 215-227 ou Rev. Peñalara. 1964, n*361, pp. 115-121.

M. Julivert, 1960 : Estudio geológico de la cuenca de Beleño (valles altos del Sella, Ponga, Nalon y Esla de la Cordillera cantábrica), Bol. IIIst. Geol. y Minero de España, t. 71, 346 p., cartes et coupes.

H. Karrenberg, 1934 : Die postvarisische Entwicklung des Kantabro-asturischen Gebirges (Nordwestspanien), publié par le C.S.I.C. in Publicaciones extranjeras sobre geologia de España, 1946, t. III, pp. 105-224.

N. Llopis-Lladó, 1950 : Sobre la tectonica germanotipica de Asturias. Real. Soc. Esp. Hist. Nat., tom. homenaje à E. Hernandez Pacheco, pp. 415-429.

N. Llopis-Lladó et L. Sole SABaris, 1954 : Geografía física, Geografia de España y Portugal (sous la direction de Manuel de Terán).

N. Llopis-Li,ADÓ, 1958 : Sobre el Karst actual y fosil de la terminación oriental de la Sierra de Cuera y sus yacimientos de hierro y manganeso, Speleón, Inst. Geol applicada, Oviedo, t. X, pp. 3-59.

N. Llopis-Lladó, 1964 : Sur la paléotectonique des Asturies et ses rapports avec la moitié occidentale de la Péninsule ibérique, Inst. Estudios Pirenaicos, Zaragoza, $n^{\circ} 4$.

J. A. Martinez-Alvarez, 1962 : Estudio geológico del reborde oriental de la cuenca carbonifera central de Asturias, Bol. I.D.E.A.

J. A. Martinez-Alvarez, 1965 : Sobre el caracter de las estructuras cabalgantes del oriente de Asturias, Notas et comunicaciones del Instituto Geol. y Minero de España, n० 78. 
L. MENGAUd, 1920 : Recherches géologiques dans la région cantabrique, Thèse Sc., Paris.

H. NonN, 1966 : Les régions côtières de la Galice (Espagne). Etude morphologique, Publ. Fac. Lettres, Strasbourg.

A.J. Pannekoek, 1959 : La sedimentación alrededor de cadenas montañosas con. ejemplos del norte de España, Geographica, Saragosse, año VI, pp. 28-24.

P. RAT, 1959 : Les pays crétacés basco-cantabriques, Thèse Sc., Paris P.U.F.

G. Schlltz, 1858 : Descripción geológica de la provincia de Oviedo, Reimpresion 1930, 176 p., Madrid.

U. DE Sitrer, 1963 : The structure of the southern slope of the Cantabrian mountains, Bol. Inst. Geol. y Minero de España, t. LXXIV, pp. 393-412.

U. De SitTer et H.J. ZWART, 1957-1958 : Voorlopige resultaten van de Kartering in Noord-Spange en Zuid-Frankvijk verkregen in 1957 door de Afdeling structurale geologie, Leidse geologische Medelingen, t. 22.

G. VIERS, 1960: Nouvelles conceptions touchant l'évolution géologique des Pyrénées, Rev. Géogr. Pyr. et Sud-Ouest, t. XXXI, fasc. 2.

\section{RES U M E}

Fragment de socle non granitisé, sédimentaire et souple, où interfèrent les tectogénèses hercyniennes et alpines; repris par une orogénèse récente (mio-pliocène ?) et vigoureuse ( $2600 \mathrm{~m}$ d'altitude). Compartimentage topographique du versant nord, proche des fosses sous-marines, conforme au jeu tectonique des blocs. Mosaïque en " écailles plissées " du versant sud, davantage solidaire de la Meseta, qui se traduit surtout indirectement par la lithologie. L'articulation des bassins-versants, qui recoupe orthogonalement les morphostructures, ne s'explique que par une mise en place synorogénique. Ebauche d'une taxonomie qui situe chaque morphostructure dans un édifice composite, massif ancien repris dans une chaîne de plissement très récente.

Morfo-estructuras cantábricas. (Picos de Europa, Montaña de León y Palencia). Fragmento de zócalo sin granitización, sedimentario $\mathrm{y}$ blando, en el cual se mezclan las estructuras hercinianas y las alpinas, modelado posteriormente por movimientos orogénicos recientes (; mio-pliocenos ?) y de gran intensidad $(2600 \mathrm{~m}$ de altitud). El recortado topográfico de la vertiente norte, cercana a las fosas submarinas, refleja la interacción tectónica de los bloques. El mosaico de " escamas plegadas " de la vertiente sur, más unido con la meseta, se manifiesta sobre todo en las formaciones líticas. La articulación de las cuencas hidrológicas en relación con la trama morfoestructural depende de una fijación, coetánea de los movimientos orogénicos. Bosquejo taxonómico que sitúa a cada morfo-estructura en un conjunto complejo, macizo herciniano parcialmente incluído en una cordillera. 
Picos de Europo et
Montagnes Contabriques Centroles

\section{LES MORPHOSTRUCTURES}

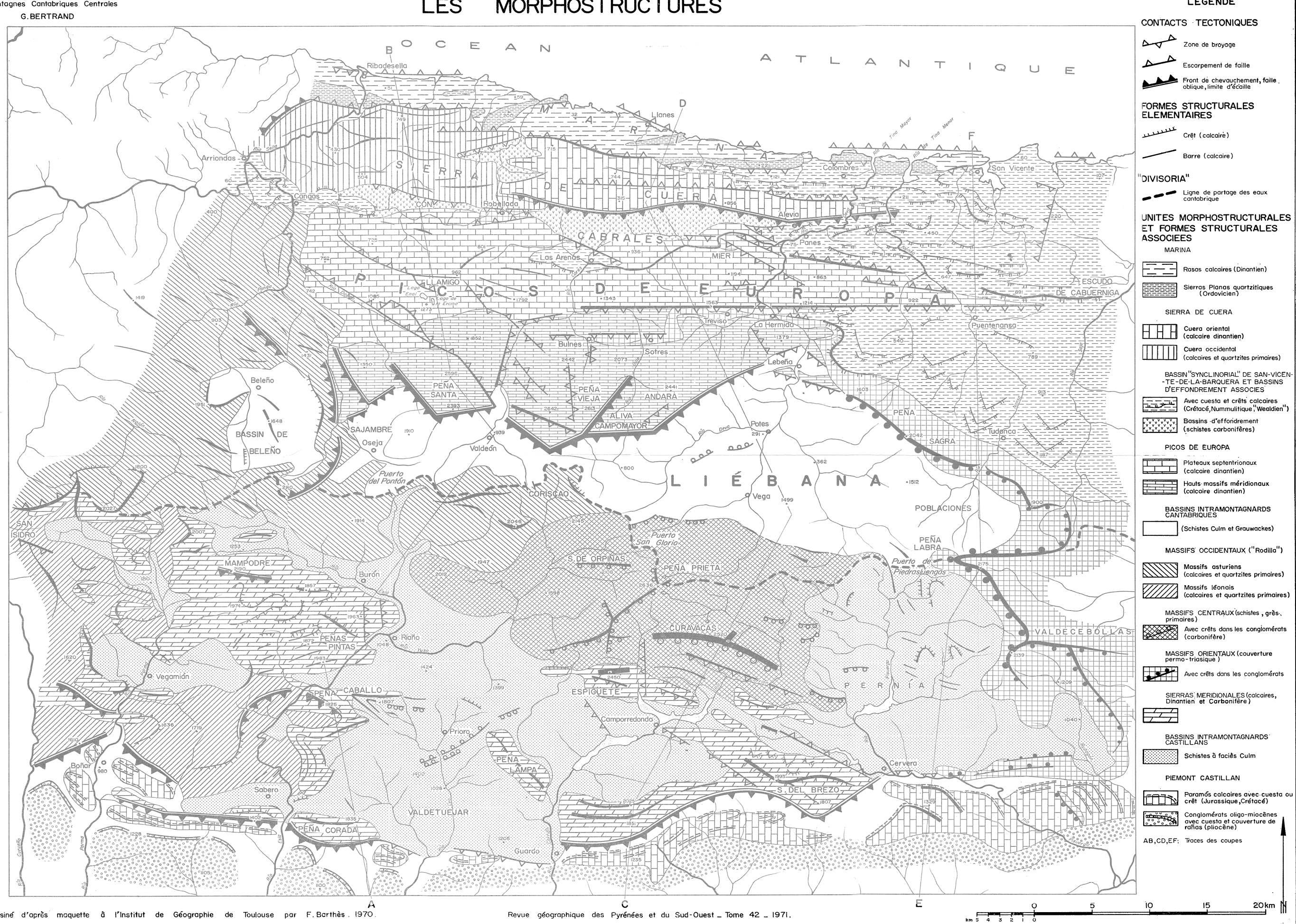



